\title{
From Genes to Behavior Through Sex Hormones and Socialization: The Example of Gender Development*
}

\author{
Sheri A. Berenbaum ${ }^{1}$ and Adriene M. Beltz ${ }^{2}$ \\ ${ }^{1}$ Departments of Psychology and Pediatrics, The Pennsylvania State University, Old Main, State College, Pennsylvania, \\ USA \\ ${ }^{2}$ Department of Psychology, University of Michigan, Ann Arbor, Michigan, USA
}

\begin{abstract}
Irving Gottesman is known primarily for his work in psychopathology, but he also had a long-standing interest in understanding psychological development generally (typical and atypical). Through his mentorship, he also influenced work in gender development. Characteristics related to sex and gender are ideally suited to study the interplay of genes and environment across development. We discuss how gender development is influenced by gonadal hormones present during early life, but not in a simple way. We describe some of the challenges and opportunities to extend our understanding of the complexity of gender development. Throughout, we consider the kinds of questions Gottesman would likely have asked and emphasize his influence on our work.
\end{abstract}

Keywords: gender development, prenatal androgens, congenital adrenal hyperplasia, geneenvironment interplay, sexual differentiation, opposite sex twins

Throughout his illustrious career, Irving (Irv) Gottesman made many contributions to our understanding of behavior. As described in this special issue and elsewhere, he is widely recognized for his development and promotion of the field of behavior genetics. Particular emphasis has been placed on his brilliant contributions in several areas (e.g., Pogue-Geile \& Lillienfeld, 2017): (a) twin studies of schizophrenia, with additional contributions to twin studies of other forms of psychopathology and personality; (b) the notion that the transmission of psychopathology is multifactorial and polygenic; (c) the related notions of diathesis-stress (individuals inherit a predisposition that results in psychopathology under triggering conditions) and reaction range (trait-specific genes set a range of phenotypes, with the specific phenotypic manifestation depending on other genes and the environment); (d) endophenotype, reflecting the pathway between genotype and disorder (i.e., a heritable anatomical, physiological, biochemical, or psychological characteristic associated with the disorder that is found even in the absence of the disorder, and seen in unaffected relatives more frequently than in the population; Gottesman \& Gould, 2003). In essence, most commemorations of Gottesman focus on his outstanding contributions to understanding psychopathology.

\section{Irv Gottesman as Developmentalist}

It is important to note that Gottesman also had a longstanding interest in understanding psychological development (typical and atypical). (His first doctoral student, Sandra Scarr, was a prominent developmentalist.) He was keen to understand the pathways (mechanisms) by which genes and environment work together to shape development. Our own work reflects this aspect of Gottesman's career, particularly in the context of gender development and sex differences, and we were touched both personally and professionally by him. One author (SAB) collaborated with him when she was a postdoctoral associate in behavioral genetics at the University of Minnesota, and they maintained communication about work on gender

RECEIVED 20 April 2018; ACCEPTEd 10 May 2018

ADDRess for Correspondence: Sheri A. Berenbaum, Departments of Psychology and Pediatrics, The Pennsylvania State University, 453 Moore, University Park, Pennsylvania, USA. E-mail: sab31@psu.edu

* The original version of this article did not include small edits, including the corresponding author address, which have now been made in the online PDF and HTML versions. 
development and the broader topic of psychological development; she also has past or current collaborations with other scientists trained by Gottesman (including Susan Resnick, Carol Prescott, and Lisabeth DiLalla), The other author (AMB) was influenced by him indirectly through her work with the first author and through collaborations with other scientists trained by Gottesman (Susan Resnick, Lisabeth DiLalla) and his students (Kelly Klump via Matthew McGue, Kristin Buss via Hill Goldsmith).

\section{Sex and Gender as Topics for Behavior Geneticists}

Characteristics related to sex and gender are ideally suited to study the interplay of genes and environment across development. The X and Y chromosomes - and the genes they carry - have major implications for anatomy and physiology, and ultimately behavior. However, sex is more than chromosomes, hormones, and genitalia. Almost all of human functioning is shaded by gender: identity, appearance, mannerisms, temperament and personality, activity interests and engagement, career choices, emotions, abilities, play and romantic partners, forms of psychopathology, aspirations, and values.

\section{Causes of Psychological Sex Differences}

The causes and consequences of being a boy or a girl have generated much scientific and public interest. Most psychological explanations focus on the role of socialization, including parent modeling and reinforcement of gendertypical behavior, and the role of children themselves in seeking out and using gender-related cues to guide their behavior. Implicit in the search for social causes of sex differences is the notion that behaviors are easier to change if they are influenced by social factors than by genetic ones, but here, as elsewhere, this is a flawed argument, as behavior geneticists, including Gottesman, have noted.

Socialization is an insufficient explanation of gender development, and there is now increasing recognition that biological predispositions play a role in gender-related characteristics as in other psychological traits. Interestingly, behavior genetic studies have rarely focused on gender-related characteristics, although studies in some domains incidentally capture them (e.g., cognitive abilities, temperament). Most genetic studies of gender-related characteristics focus on the effects of genes on the sex chromosomes, often studied in people with sex chromosomal anomalies (e.g., the role of the $\mathrm{X}$ chromosome in spatial ability studied in women with Turner syndrome). (For review and discussion, see Blakemore et al., 2009.)

Most work on biological contributors to sex-related characteristics focuses on gonadal hormones present during sensitive periods of development, particularly prenatal life. Many studies in a variety of non-human species provide converging and compelling evidence that androgens and estrogens present early in development affect sexual differentiation of many aspects of behavior, as well as reproductive anatomy and function (reviewed in de Vries et al., 2014; Wallen, 2009). (There is increasing interest in early infancy, puberty, and pregnancy as other sensitive periods for the effects of androgens and estrogens, but this topic is beyond the scope of our paper; for discussion, see Berenbaum \& Beltz, 2016.)

\section{Early Hormonal Influences on Gender Development}

It is now clear that early development represents a sensitive period for hormones (particularly androgens) in the organization of the human brain and behavior. Most evidence comes from individuals with a disorder of sex development (DSD), where there is discordance among levels of physical sexual differentiation. Converging evidence is beginning to come from typical samples; as noted below, this is an area of great research opportunity.

Most of what we know about the psychological effects of early hormones comes from females with the most common DSD, classical congenital adrenal hyperplasia (C-CAH). C$\mathrm{CAH}$ is inherited in an autosomal recessive fashion, and is caused by a genetic mutation (in CYP21A) that results in exposure to excess androgens beginning early in gestation. Affected individuals are usually diagnosed and treated in the early newborn period, so generally have low postnatal androgens, and females with $\mathrm{C}-\mathrm{CAH}$ are reared as girls. Thus, females with C-CAH provide a separation of hormonal sex (masculinized relative to unaffected females) and social sex (typical female). To examine the psychological effects of exposure to excess prenatal androgens, females with $\mathrm{C}-\mathrm{CAH}$ are generally compared to unaffected females (optimally their sisters without $\mathrm{C}-\mathrm{CAH}$ ) and occasionally to males with and without $\mathrm{C}-\mathrm{CAH}$. An interesting comparison is provided by females with a mild form of the disorder, non-classical (NC) CAH, who are exposed to excess androgens in the postnatal period only, usually starting in midchildhood or adolescence (Merke \& Bornstein, 2005); these cases provide a control for disease and treatment.

Studies in females with CAH show the complexity of androgen effects on behavior (for details, discussion, and references, see Berenbaum, 2018; Berenbaum \& Beltz, 2011, 2016). For many personal characteristics, androgen effects track sex differences, with androgen effects generally smaller in size than sex effects. Androgens masculinize activity interests: toy play in childhood, activity interests and engagement in adolescence, hobbies and leisure pursuits in adulthood. Androgen effects extend to occupational interests, especially interest in occupations involving things versus people, and to actual occupational choice. Androgens masculinize personal-social characteristics, including interest in babies and aggression. Androgens masculinize cognitive abilities, especially spatial and 
mechanical abilities; Gottesman was an author on one of the first papers on spatial enhancement in females with $\mathrm{CAH}$, in a study headed by Susan Resnick, one of his graduate students (Resnick et al., 1986).

However, moderate levels of androgens during prenatal life have minimal effects on gender identity and gender cognitions. The overwhelming majority of girls and women with $\mathrm{CAH}$ are female-identified, although their degree of identification is somewhat lower than that of unaffected females, and the small minority ( $\sim 5 \%)$ of females with $\mathrm{CAH}$ who are gender dysphoric or identify as male is greater than that in the general population. Females with CAH also have typical gender attitudes and ideas about gender.

Differential androgen effects have been replicated across age, method, and country and, as such, different genderrelated behaviors have different causes. But these differential effects do more than describe sources of behavioral variation; they also help delineate developmental pathways. This is illustrated in data on peer interactions in girls with CAH (Berenbaum et al., 2018). Time spent with girls (reported in daily phone calls across seven days) was not significantly related to the degree of prenatal androgen exposure, but was predicted by both interest and engagement in gender-typical activities and gender identity/cognitions.

\section{Pathways From Genes to Behavior Through Androgens}

As Irv would undoubtedly ask, so now what? How do we move from asking whether androgens affect human behavior to asking how they do so? Here are some questions and opportunities that might delineate the role of early androgen exposure in the path from genes to behavior. First, what are the neural substrates underlying androgen effects on behavior? It is important to remember that the brain is the synthesis of biology and socialization; experience changes the brain. Much work is directed to describing sex differences in brain structure and task activation, and neural 'signatures' could be considered endophenotypes for gender-related psychopathology; it is important to remember, however, that those differences might cause or result from psychological sex differences. Therefore, studies of hormonal influences on neural sex differences must also include behavioral measures and consider the dynamic nature of brain structure (for discussion, see Beltz et al., 2013).

Second, what are the behavioral mechanisms underlying androgen effects on behavior? For example, why does prenatal exposure to high levels of androgens lead someone to prefer and engage with mechanical activities, or to pursue engineering as a career? Intriguingly, the gendered toy preferences of children are paralleled in rhesus monkeys (Hassett et al., 2008). These differences may be driven, in part, by sex differences in predisposition to imitate propulsive movement (hitting vs. cradling an object; Benenson et al.,
2011), and by preferences for engagement with people versus objects (Beltz et al., 2011).

Third, how do androgen-influenced predispositions affect a person's transactions with the social environment? We know shockingly little about such hormone-environment correlations and interactions, although this is probably the most compelling question because it speaks to mechanism.

\section{Opportunities to Understand How Hormones Influence Gender Development}

Answers to the questions raised above will help us to understand how biological predispositions become enacted over development, a topic of keen interest to Irv. In pursuing this work, it is essential to extend the findings from individuals with DSD to the general population.

There are methods for studying prenatal androgen effects on behavior in the general population, but they have limitations. One method, linking amniotic hormones to childhood behavior, involves only a single sample of hormones during gestational periods that vary across participants, and includes women who undergo amniocentesis and are, therefore, not representative of the general population.

Other methods include purported biomarkers of prenatal androgen exposure. Biomarkers are often easy to obtain, so are commonly used, but most have limited value because they are not validated.

The most popular biomarker, the relative length of the second to fourth fingers (known as $2 \mathrm{D}: 4 \mathrm{D}$ or digit ratio) is not a good indicator of individual differences in prenatal androgen levels, as explained below. The most compelling evidence comes from individuals with complete androgen insensitivity syndrome (CAIS) who have a male-typical 46,XY karyotype with normal testes and male-typical levels of testosterone, but absent or dysfunctional androgen receptors that leave them unable to respond to endogenous or exogenous androgens (Berenbaum et al., 2009). As expected from their reduced prenatal androgen exposure, they have feminized (high) digit ratios, although the difference with typical men is only moderate in size; therefore, digit ratio is not a good predictor of group membership (CAIS, typical women, typical men). Crucially, individuals with CAIS show as much variability in digit ratio as do typical men and women, despite having no variability in effective androgen exposure. So - in line with Irv's reminders to use data to inform our work - it is (past) time to stop using digit ratio as a marker of prenatal androgen exposure.

Fortunately, there is another potential method for extending the findings from individuals with DSD to the general population that takes advantage of the expertise and resources of twin researchers: opposite-sex twins. Data from non-human animals show that uterine position during gestation influences various aspects of 


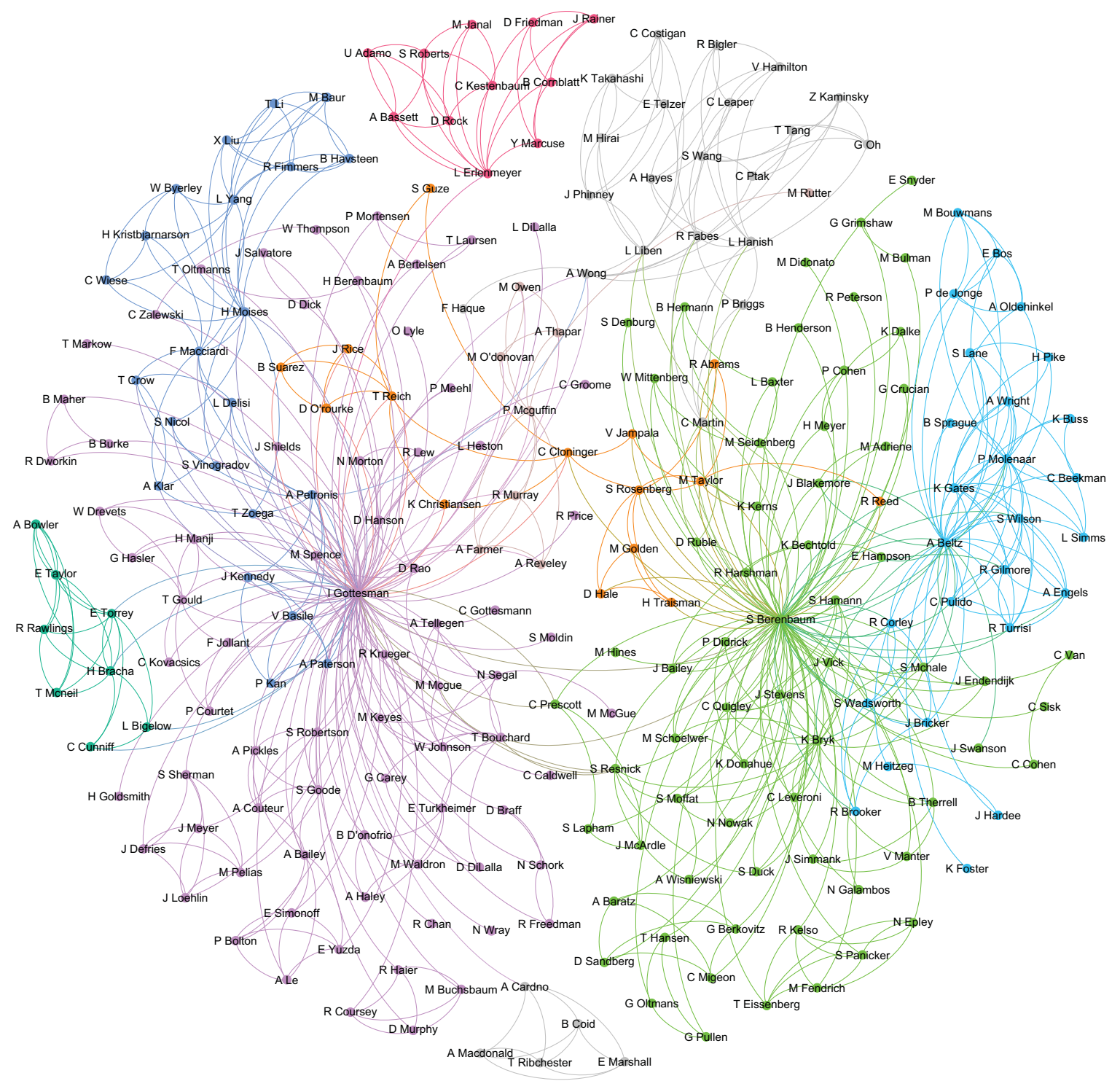

FIGURE 1

(Colour online) Combined collaborator networks for Irving Gottesman and the authors of this paper (S. Berenbaum and A. Beltz), illustrating the extent of Gottesman's direct and indirect influence on the field of psychology. Networks are based on select publications from Google Scholar profiles as of April 2018 (Barbera, 2016; Bastian et al., 2009).

anatomy, physiology, and behavior (Ryan \& Vandenbergh, 2002). Female rodents that gestate between two males are masculinized compared to females that gestate between two females; females next to one male show some masculinization. Similar effects have been seen in other species, including swine. The effect is due to the transfer of testosterone from male fetuses to adjacent female fetuses, although the exact mechanism remains to be specified (e.g., relative role of diffusion vs. transfer through blood).

A key question is whether this phenomenon operates in human beings, and Irv was involved in the first study to address this question; he and his colleagues found that females with a male co-twin scored higher in sensation seeking than females with a female co-twin (Resnick et al., 1993). Sub- sequent studies of behavioral masculinization in oppositesex twins are inconsistent, with some revealing that females with a male co-twin are masculinized and/or defeminized compared to females with a female co-twin, and some failing to find effects of co-twin sex (for review and discussion, see Tapp et al., 2011).

Much work remains to be done to determine the value of this natural experiment for studying the behavioral effects of prenatal androgens. Perhaps the most pressing need is for validity: Are females with a male co-twin exposed to higher levels of androgens in utero than females with a female co-twin? The answer might best be obtained by examining anogenital distance in newborn twins, because this anatomical measure is sensitive to prenatal androgens 
(Thankamony et al., 2016). And, then, it would be worthwhile to consider factors that might moderate the effect of androgen exposure due to uterine position. This includes factors unique to multiple births, such as the degree of uterine sharing, the potential effect of the number of opposite-sex siblings (twins vs. triplets, in light of animal studies that show bigger effects for gestating between two opposite-sex littermates than for gestating next to only one), and factors that operate in singletons as well, such as variations in androgen responsivity (e.g., androgen receptor gene). Furthermore, it is essential to include comparisons for postnatal socialization (being reared with an opposite-sex sibling), and to consider whether opposite-sex twin effects vary across behaviors, as they do in females with CAH (as discussed above). Ultimately, if (at least some) females with a male co-twin are found to be exposed to aboveaverage androgens during prenatal life, they can help us to understand the neural and behavioral mechanisms that underlie those effects, and how hormones and socialization work together to produce behavioral development.

\section{Irv Gottesman's Legacy}

We hope that Gottesman's influence on our careers and thinking has been apparent in this article. For the first author, the postdoc in behavioral genetics at Minnesota provided the opportunity and impetus to study people with $\mathrm{CAH}$, and her work there and subsequent work (including with the second author and with other scientists trained by Irv) were facilitated by Irv's collaboration, mentoring, and support. He encouraged her critical thinking, her desire to understand the complexity of behavioral development, and the importance of using our science to benefit society. Through him, she met smart and interesting people who became friends and colleagues. For the second author, her research to uncover mechanisms underlying gender development has been powered by the use of natural experiments (such as CAH, opposite-sex twins, and in her independent work, exogenous hormones via oral contraceptive use), a direction that might never have emerged had Irv not encouraged the first author to study CAH in Minnesota. Our own collaboration illustrates the importance and joy Irv emphasized in mentoring and in training future generations.

Irv's influence on us is a piece of a much larger picture, as seen in Figure 1, that displays our combined authorship-based collaborator networks; it shows Gottesman's extensive links to other scientists, including both of us and the authors of papers in this special issue. The figure also reveals the breadth of Gottesman's influence and his ability to cultivate collaborations: Networks like this exist for his 100+ co-authors; and many of his collaborators went on to conduct independent collaborations with each other. These links represent the strong and rewarding personal and professional relationships formed between Irv and all of us. Time with Irv was always engaging and stimulating, and he made us all better scientists and people.

\section{Acknowledgments}

Our research reported here was supported by grants from the United States National Institutes of Health, HD19644, HD057930, HD044398, and MH099617. We appreciate the many people who have contributed to the research, as noted in the publications describing the work. For especially significant help, we thank Kristina Bryk who skillfully managed the projects investigating psychological functioning in people with $\mathrm{CAH}$, and who contributed to all stages of the work from conceptualization through dissemination; collaborators, particularly Susan McHale; pediatric endocrinologists and family support group staff who assisted with the recruitment of families; and graduate and undergraduate students, and research assistants who helped to coordinate studies, and collect and process data. We are especially grateful to the families who participated in these studies.

\section{References}

Barbera, P. (2016). Extract and visualize Google Scholar collaboration networks. GitHub Repository. Retrieved from https://github.com/pablobarbera/scholarnetwork.

Bastian, M., Heymann, S., \& Jacomy, M. (2009, May). Gephi: An open source software for exploring and manipulating networks. Paper presented at the International AAAI Conference on Weblogs and Social Media, San Jose, CA.

Beltz, A. M., Blakemore, J. E. O., \& Berenbaum, S. A. (2013). Sex differences in brain and behavioral development. In P. Rakic \& J. Rubenstein (Eds.), Comprehensive developmental neuroscience (vol. 3, pp. 467-499). Oxford, UK: Elsevier.

Beltz, A. M., Swanson, J. L., \& Berenbaum, S. A. (2011). Gendered occupational interests: Prenatal androgen effects on psychological orientation to Things versus People. Hormones and Behavior, 60, 313-317.

Benenson, J. F., Tennyson, R., \& Wrangham, R. W. (2011). Male more than female infants imitate propulsive motion. Cognition, 121, 262-267.

Berenbaum, S. A. (2018). Beyond pink and blue: The complexity of early androgen effects on gender development. Child Development Perspectives, 12, 58-64.

Berenbaum, S. A., \& Beltz, A. M. (2011). Sexual differentiation of human behavior: Effects of prenatal and pubertal organizational hormones. Frontiers in Neuroendocrinology, 32, 183-200.

Berenbaum, S. A., \& Beltz, A. M. (2016). How early hormones shape gender development. Current Opinion in Behavioral Sciences, 7, 53-60.

Berenbaum, S. A., Beltz, A. M., Bryk, K. L., \& McHale, S. M. (2018). Gendered peer involvement in girls with congenital adrenal hyperplasia: Effects of prenatal androgens, gendered activities, and gender cognitions. Archives of Sexual Behavior, 47, 915-929. 
Berenbaum, S. A., Bryk, K. K., Nowak, N., Quigley, C. A., \& Moffat, S. (2009). Fingers as a marker of prenatal androgen exposure. Endocrinology, 150, 5119-5124.

Blakemore, J. E. O., Berenbaum, S. A., \& Liben, L. S. (2009). Gender development. New York, NY: Psychology Press/Taylor \& Francis.

de Vries, G. J., Fields, C. T., Peters, N. V., Whylings, J., \& Paul, M. J. (2014). Sensitive periods for hormonal programming of the brain. In S. L. Andersen \& D. S. Pine ( Eds.), Current topics in the behavioral neurosciences: The neurobiology of childhood (vol. 16, pp. 79-108). Berlin: Springer.

Gottesman, I. I., \& Gould, T. D. (2003). The endophenotype concept in psychiatry: Etymology and strategic intentions. American Journal of Psychiatry, 16, 636-645.

Hassett, J. M., Siebert, E. R., \& Wallen, K. (2008). Sex differences in rhesus monkey toy preferences parallel those of children. Hormones and Behavior, 54, 359-364.

Merke, D. P., \& Bornstein, S. R. (2005). Congenital adrenal hyperplasia. Lancet, 365, 2125-2136.

Pogue-Geile, M. F., \& Lillienfeld, S. O. (2017). Irving I Gottesman (1930-2016): In memoriam - Introduction to the special section. Clinical Psychological Science, 5, 418-419.
Resnick, S. M., Berenbaum, S. A., Gottesman, I. I., \& Bouchard, T. J. (1986). Early hormonal influences on cognitive functioning in congenital adrenal hyperplasia. Developmental Psychology, 22, 191-198.

Resnick, S. M., Gottesman, I. I., \& McGue, M. (1993). Sensation-seeking in opposite-sex twins: An effect of prenatal hormones? Behavior Genetics, 23, 323-329.

Ryan, B. C., \& Vandenbergh, J. G. (2002). Intrauterine position effects. Neuroscience and Biobehavioral Reviews, 26, 665678.

Tapp, A. L., Mayberry, M. T., \& Whitehouse, A. J. (2011). Evaluating the twin testosterone transfer hypothesis: A review of the empirical evidence. Hormones and Behavior, 60, 713722.

Thankamony, A., Pasterski, V., Ong, K. K., Acerini, C. L., \& Hughes, I. A. (2016). Anogenital distance as a marker of androgen exposure in humans. Andrology, 4, 616-625.

Wallen, K. (2009). The organizational hypothesis: Reflections on the 50th anniversary of the publication of Phoenix, Goy, Gerall, and Young (1959). Hormones and Behavior, 55, 561565. 